\title{
Endoscopic Band Ligation for Acute Lower Gastrointestinal Bleeding
}

\author{
Yasutoshi Shiratori ${ }^{1}$, Takashi Ikeya ${ }^{1}$, Naoki Ishii ${ }^{2}$, Ayaka Takasu ${ }^{1}$, Hirokazu Honda ${ }^{1}$, \\ Kenji Nakamura ${ }^{1}$ and Katsuyuki Fukuda ${ }^{1}$
}

\begin{abstract}
:
Objective Endoscopic band ligation (EBL) is commonly performed to treat colonic diverticular bleeding (CDB). However, EBL is not suitable for other disorders that cause acute lower gastrointestinal bleeding (ALGIB), and the safety and efficacy of the procedure are not well known. This study aimed to evaluate the efficacy and safety of EBL for non-colonic diverticular bleeding (non-CDB) and investigate the application of EBL to ALGIB.

Methods This study was a retrospective evaluation of the success rate of EBL, the rate of early re-bleeding (within 30 days of the initial EBL), and complications such as perforation and abscess formation.

Patients Thirty patients who presented with non-CDB and underwent EBL as the first-line treatment in our hospital from June 2009 to December 2017 were included in the present study.

Results The success rate of EBL was 93\% (28/30). The rate of early re-bleeding after EBL was 20\% (6/ 30). Repeat EBL, endoscopic clipping, or conservative therapy was performed in the event of re-bleeding. No emergency surgery or interventional hemostatic treatments were required for hemostasis. No complications such as perforation or abscess formation were observed in any patient.

Conclusion Our results suggest that EBL is an effective and safe endoscopic treatment for non-CDB.
\end{abstract}

Key words: endoscopic band ligation, acute lower gastrointestinal bleeding, endoscopic hemostasis, acute hemorrhagic rectal ulcer, therapeutic endoscopy

(Intern Med 58: 3505-3508, 2019)

(DOI: 10.2169/internalmedicine.3185-19)

\section{Introduction}

Acute lower gastrointestinal bleeding (ALGIB) is a common disease, which sometimes leads to hemorrhagic emergency (1). Colonic diverticular bleeding (CDB) is a common cause of ALGIB, and endoscopic band ligation (EBL) has been recommended as a treatment for CDB due to its safety, efficacy, and long-term results (2-4). Specifically, in terms of the rebleeding rate, EBL may be superior to other hemostasis methods, including clipping (5). However, the usefulness of EBL in other disorders that result in ALGIB is limited, and the safety and efficacy of EBL for treating non-CDB are not well established. In this study, we examined the hemostatic results in non-CDB treated by EBL over the past 8 years and evaluated the efficacy and safety of this procedure.

\section{Materials and Methods}

Fig. 1 shows a flow chart of the present study. Colonoscopy was performed in 789 patients who presented with ALGIB at St. Luke's International Hospital in Tokyo, Japan between June 2009 and December 2017. We excluded presumptive cases, which were considered to be without stigmata of recent hemorrhage (SRH) in colonic endoscopy (as defined by Jensen et al.) (6). Of the 372 definitive cases, patients who did not require hemostatic treatment-such as those presenting with ischemic enteritis and colorectal cancer or those who had previously been treated with a non-

${ }^{1}$ Department of Gastroenterology, St. Luke's International Hospital, Japan and ${ }^{2}$ Department of Gastroenterology, Tokyo Shinagawa Hospital, Japan

Received for publication April 15, 2019; Accepted for publication July 3, 2019

Correspondence to Dr. Yasutoshi Shiratori, shiraya@luke.ac.jp 
EBL method-were excluded. Furthermore, patients diagnosed with $\mathrm{CDB}$ were excluded from this study. A total of 30 patients (20 men and 10 women; age: 40-101 years) received EBL for non-CDB. EBL was selected as a hemostasis method in non-CDB when bleeding could not be stopped by other methods such as endoscopic clipping, heat coagulation, or epinephrine injection (1:20,000 dilution). The origin of bleeding was identified as the site at which SRH was observed, and SRH was defined as active bleeding (AB), a densely adherent clot, and a non-bleeding visible vessel (NBVV) (6). Early re-bleeding was defined as clinical evidence of recurrent lower gastrointestinal bleeding within 30 days of the initial treatment. The etiologies of the cases in the present study were as follows: acute hemorrhagic rectal ulcer (AHRU; $n=17$ ), diverticular bleeding in the small intestine $(n=5)$, Dieulafoy's ulcer in the small intestine and post-polypectomy bleeding $(n=2)$, rectal varices $(n=2)$, postprostate biopsy $(n=1)$ and post-appendectomy bleeding $(n=$ $1)$.

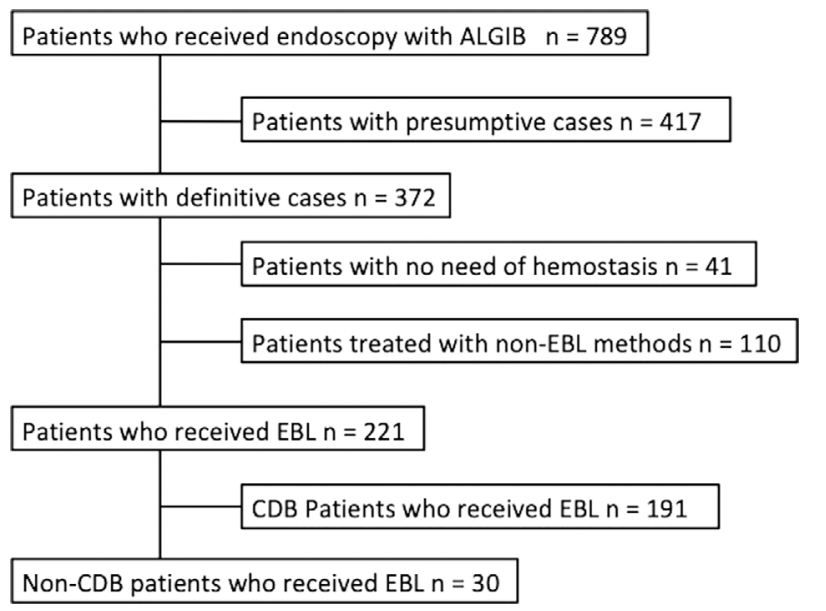

Figure 1. Diagram of the study flow. ALGIB: acuter lower gastrointestinal bleeding, CDB: colonic diverticular bleeding, EBL: endoscopic-band ligation
We retrospectively evaluated the success rate of EBL, the rate of early re-bleeding, the period from the initial treatment to re-bleeding, and complications including perforation and abscess formation.

This study was approved by our institutional review board. We received approval from the clinical ethics committee for the use of an EBL device for non-CDB and the patients directly gave their informed consent prior to hemostatic treatment.

\section{Band ligation technique}

After admission, bowel preparation with polyethylene glycol was performed. Colonoscopy was performed using a water-jet-scope (PCFQ260JI or PCF290I, PCF260AZI; Olympus Optical, Tokyo, Japan). Once the sources of bleeding were identified (Fig. 2A), they were marked with hemoclips (HX-610-135; Olympus Optical) near the origin of bleeding. Subsequently, the endoscope was removed and reinserted with the band-ligator device attached (MD-48710 EVL Device; Sumitomo Bakelite, Tokyo, Japan). We used a band-ligator device that was similar to that used for the treatment of esophageal varices. The bleeding site was suctioned into the band ligator, and the elastic O-band was released (Fig. 2B). Marking with hemoclips was not performed in the case of post-polypectomy bleeding (Fig. 3).

Where early re-bleeding occurred, colonoscopy was performed to identify the source of re-bleeding, and additional treatments such as repeat EBL, endoscopic clipping, heat coagulation, and epinephrine injection were performed as necessary.

\section{Statistical analysis}

Statistical analysis was performed using the $\mathrm{JMP}^{\circledR}$ software program (version 14, SAS Institute, Cary, USA). The patients' ages are reported as the median, and the success rate of EBL and rate of early re-bleeding are presented as the proportion and $95 \%$ confidence interval $(95 \% \mathrm{CI})$.

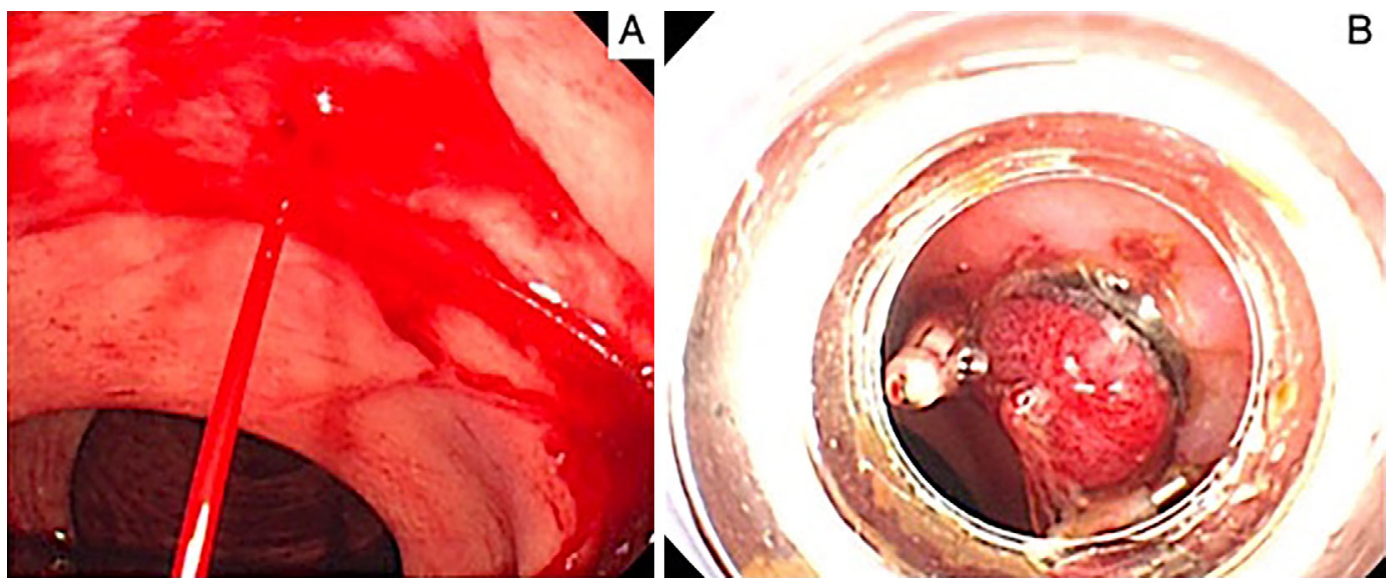

Figure 2. (A) An endoscopy image showing a small rectal ulcer with spurting bleeding. (B) After marking with a hemoclip near the ulcer, endoscopic-band ligation was performed. Hemostasis was obtained immediately after endoscopic band ligation. 


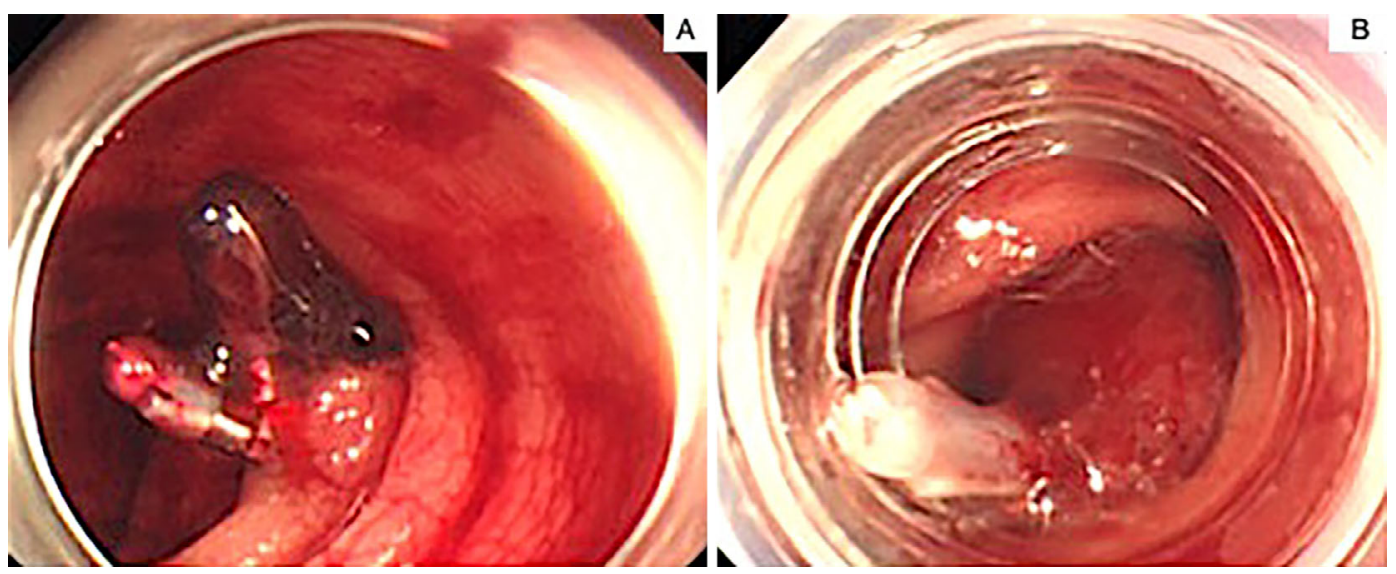

Figure 3. (A) An endoscopy image showing post-polypectomy bleeding after preventive clipping. (B) An image of endoscopic band ligation performed for the treatment of post-polypectomy bleeding.

Table. The Procedural Details and Outcomes.

\begin{tabular}{|c|c|c|c|c|c|c|}
\hline & Case & Age & Male/Female & Location & Success rate $(\%)$ & Rebleed rate $(\%)$ \\
\hline AHRU & 17 & 83.5 & $11 / 6$ & $\mathrm{R} 17$ & 88 & 29 \\
\hline Diverticular bleeding in small intestine & 5 & 65 & $3 / 2$ & J2, IL3 & 100 & 20 \\
\hline Dieulafoy's ulcer in small intestine & 2 & 80.5 & $0 / 2$ & J1, IL1 & 100 & 0 \\
\hline Postpolypectomy bleeding & 2 & 61.5 & $2 / 0$ & $\mathrm{~A} 2$ & 100 & 0 \\
\hline Rectal varices & 2 & 90 & $2 / 0$ & $\mathrm{R} 2$ & 100 & 0 \\
\hline Postappendectomy bleeding & 1 & 40 & $1 / 0$ & $\mathrm{C} 1$ & 100 & 0 \\
\hline Postprostate biopsy bleeding & 1 & 87 & $1 / 0$ & R1 & 100 & 0 \\
\hline Total & 30 & 83 & $20 / 10$ & $\mathrm{~J} 3, \mathrm{IL} 4, \mathrm{C} 1, \mathrm{~A} 2, \mathrm{R} 20$ & 93.3 & 20 \\
\hline
\end{tabular}

A: ascending colon, AHRU: acute hemorrhagic rectal ulcer, C: cecum, IL: ileum, J: jejunum, R: rectum

\section{Results}

The EBL results are summarized in Table. The overall success rate of EBL for non-CDB was $93.3 \%$ (95\% CI: 0.82-1.03). AHRU was the most common cause of non-CDB that was treated with EBL, followed by Dieulafoy's ulcer in the small intestine. EBL was reported to be unsuccessful for two cases of AHRU. The rate of early re-bleeding was $20 \%$ (95\% CI: 0.05-0.38). The rate of early re-bleeding was $29.4 \%$ and $20 \%$ in cases of AHRU and small intestinal diverticular bleeding, respectively. The period from the initial treatment to re-bleeding ranged 3-11 days (median 7 days). The average treatment time from the identification of the source of hemorrhage to hemostasis was 18 minutes.

Neither emergency surgery nor interventional hemostatic treatment was required in any case. No complications such as perforation or abscess formation were observed in any patient during a mean follow-up period of 26 months.

\section{Discussion}

The use of EBL as a treatment for CDB has been established through other retrospective studies $(7,8)$. The present retrospective study analyzed the results of EBL performed in 30 cases of definite hemorrhage where the site of bleeding was identified. Our results revealed that EBL has a high success rate and that it can safely be applied in the treatment of non-CDB.

The median age of the subjects in this study was 83.5 years, and AHRU was found to develop in elderly people with decreased activities of daily living. Two cases of unsuccessful EBL were reported. In these cases, the exposed vessel had a hard ulcer and could not be suctioned into the cup of the band-ligator. In such cases, endoscopic clipping or epinephrine injection was performed, and a high rate of rebleeding $(29 \%, 5 / 17$ cases) was seen. This may be associated with the intake of multiple drugs, such as antiplatelet or anticoagulant medications, for disorders such as cerebral infarction and arrhythmia. Furthermore, some patients with AHRU present with multiple lesions, which may be related to re-bleeding (9). Thus, the re-bleeding may not have been a result of EBL-rather, it might have been related to AHRU. One case report suggested that EBL is effective despite rebleeding after the application of the clip method for AHRU (10). It would therefore be beneficial to compare EBL with other modalities including clipping in future studies.

EBL was successfully performed for lesions of the small intestine in all of 7 cases (three cases of ileal diverticular bleeding, two cases of ileal Dieulafoy's ulcer, and one case each of jejunal diverticular bleeding and jejunal Dieulafoy's ulcer). Double-balloon enteroscopy was performed for the treatment of the two cases of jejunal bleeding. Early re- 
bleeding occurred in one case, when dislodgement of the $\mathrm{O}$ band occurred. The walls of the small intestine are thin, and there is a risk of perforation due to the absence of a muscle layer in the diverticula (11); however, no complications or instances of perforation or penetration were observed in the cases of the present study. Although we only evaluated seven cases, our results suggest that EBL can be considered a safe treatment for small intestinal bleeding as well as CDB.

Two cases of post-polypectomy bleeding were successfully treated with EBL with no complications. Despite the use of hemoclips at the time of polypectomy, oozing continued, and we could suction the lesion with the hemoclips into the band ligator and apply EBL to achieve hemostasis of these lesions. This could have ligated the culprit vessels and hence prevented further bleeding. A case report showed the efficacy of EBL for post-polypectomy bleeding (12).

The two patients with rectal varices had liver cirrhosis due to hepatitis $\mathrm{C}$ infection or alcohol use. Ectopic varices including rectal varices are caused by portal hypertension (13). In these cases, bleeding was successfully treated with EBL and no early re-bleeding was observed. Although there have been retrospective studies on hemostasis for ectopic varices, including rectal varices (14), there is no definitive hemostatic method for rectal varices. EBL should be attempted for such cases in the future.

Bleeding following prostate biopsy is rare but can be massive at times (15). In the single case included in this study, visualization was difficult due to massive bleeding. The origin of the bleeding was difficult to identify, and EBL was performed at the suspected source. In such cases, EBL might be useful because it is not a complex procedure, and banding is achieved merely by pressing the device against the source of bleeding.

Post-appendectomy active bleeding occurred in the deep part of the appendicular opening, and so the origin of the bleeding could not be confirmed. Thus, EBL was considered more effective than hemoclip placement in this context.

The present study was associated with several limitations. First, this was a retrospective study from a single center. Second, the study included a relatively small number of cases. Third, hemostatic method that was applied was based on the judgment of the endoscopist. The results presented here are encouraging and warrant further prospective trials, involving larger numbers of patients with a longer follow-up period.

In conclusion, we performed EBL for non-CDB due to various causes. Our results indicate that EBL may be an ef- fective and safe endoscopic treatment for non-CDB.

The authors state that they have no Conflict of Interest (COI).

\section{References}

1. Farrar FC. Management of acute gastrointestinal bleed. Crit Care Nurs Clin North Am 30: 55-66, 2018.

2. Witte JT. Band ligation for colonic bleeding: modification of multiband ligating devices for use with a colonoscope. Gastrointest Endosc 52: 762-765, 2000.

3. Farrell JJ, Graeme-Cook F, Kelsey PB. Treatment of bleeding colonic diverticula by endoscopic band ligation: an in-vivo and exvivo pilot study. Endoscopy 35: 823-829, 2003.

4. Ishii N, Setoyama T, Deshpande GA, et al. Endoscopic band ligation for colonic diverticular hemorrhage. Gastrointest Endosc 75: 382-387, 2012.

5. Nakano K, Ishii N, Ikeya T, et al. Comparison of long-term outcomes between endoscopic band ligation and endoscopic clipping for colonic diverticular hemorrhage. Endosc Int Open 3: E529-E $533,2015$.

6. Jensen DM, Machicado GA, Jutabha R, Kovacs TO. Urgent colonoscopy for the diagnosis and treatment of severe diverticular hemorrhage. New Engl J Med 342: 78-82, 2000.

7. Marques S, Barreiro P, Chagas C. Endoscopic band ligation: a safe and effective treatment for active diverticular bleeding. ACG Case Rep J 3: 77-78, 2016.

8. Shimamura Y, Ishii N, Omata F, et al. Endoscopic band ligation for colonic diverticular bleeding: possibility of standardization. Endosc Int Open 4: E233-E237, 2016.

9. Maneerattanaporn M, Pongpaibul A, Pongprasobchai S, Kachintorn U, Manatsathit S. Acute hemorrhagic rectal ulcer syndrome: the first case series from Thailand. J Med Assoc Thai 95 (Suppl 2): S48-S55, 2012.

10. Yoshikumi Y, Mashima H, Suzuki J, et al. A case of rectal Dieulafoy's ulcer and successful endoscopic band ligation. Can J Gastroenterol 20: 287-290, 2006.

11. Ikeya T, Ishii N, Shimamura Y, et al. Endoscopic band ligation for bleeding lesions in the small bowel. World J Gastrointest Endosc 6: 488-492, 2014.

12. Smith RE, Doull J. Treatment of colonic post-polypectomy bleeding site by endoscopic band ligation. Gastrointest Endosc 40: 499500, 1994.

13. Lebrec D, Benhamou JP. Ectopic varices in portal hypertension. Clinics in gastroenterology 14: 105-121, 1985.

14. Watanabe N, Toyonaga A, Kojima S, et al. Current status of ectopic varices in Japan: results of a survey by the Japan Society for Portal Hypertension. Hepatol res 40: 763-776, 2010.

15. Strate LL, O'Leary MP, Carr-Locke DL. Endoscopic treatment of massive rectal bleeding following prostate needle biopsy. Endoscopy 33: 981-984, 2001.

The Internal Medicine is an Open Access journal distributed under the Creative Commons Attribution-NonCommercial-NoDerivatives 4.0 International License. To view the details of this license, please visit (https://creativecommons.org/licenses/ by-nc-nd/4.0/).

(C) 2019 The Japanese Society of Internal Medicine Intern Med 58: 3505-3508, 2019 